\title{
Wireless Monitoring and Remote Control of PV Systems Based on the ZigBee Protocol
}

\author{
V. Katsioulis ${ }^{1}$, E. Karapidakis ${ }^{2}$, M. Hadjinicolaou ${ }^{1}$, and A. Tsikalakis ${ }^{2}$ \\ ${ }^{1}$ School of Engineering and Design, Brunel University, UB8 3PH, Uxbridge, UK \\ ${ }^{2}$ Renewable Energy Engineering Lab, TEIC, Romanou 3 str, 73133 Chania, Greece
}

\begin{abstract}
Systems that convert the sunlight into electrical energy like photovoltaics (PV) have been becoming widespread worldwide. The prospect of using the promising technology of wireless sensor networks (WSN) in the field of PV plant supervising and monitoring is studied here. The knowledge of the status and good working condition of each PV module separately as well as of any PV system component will lead in a more efficient way for power management. The nature of the wireless sensor networks (WSN) offers several advantages on monitoring and controlling applications over other traditional technologies including self-healing, self-organization and flexibility. The versatility, ease of use and reliability of a mesh network topology offered by the ZigBee technology that is based on the IEEE 802.15.4 standard, is used here to offer its maximum advantages on a system that is capable for real time measurements and event alerts.
\end{abstract}

Keywords: Photovoltaic panels, PV monitoring \& control, wireless sensors networks and ZigBee.

\section{Introduction}

Many monitoring (data-acquisition) systems have been proposed and developed. The main task of these systems is to monitor and collect data concerning the performance of the PV plant in real time. In [1] a system for remote monitoring and control of complex stand-alone photovoltaic plants is proposed. It is based on the NI Field-Point architecture, an FP-2000 controller and an FP-I-100 acquisition system. Current, voltage and temperature data are transmitted using a GSM modem. A different approach has been proposed in [2], [3] where a computer-based data-acquisition system monitors both electrical and meteorological data. Remote clients can reach data using the TCP/IP protocol. Another proposal of PV monitoring is described in [4].

The above proposals give only a general performance monitoring image. They do not provide information about the performance and state of each individual PV module. In some cases, a specific PV module in a large scale PV plant may produce no or lower energy levels than expected. In this case, in a monitoring system architecture similar with those described above, the system will be able to sense and monitor the lower current or voltage reading but it has no ability to locate the source of the problem. This is mainly because current and voltage sensors are connected at 
the output of a PV array that might be part of several PV modules. In this case it is obvious that a maintenance operator is needed to manually locate the defective part.

Wireless sensor networks (WSN) are a very promising technology in the field of PV monitoring. A wireless sensor network is a system which comprises radio frequency (RF) transceivers, sensors, micro-controllers and power sources. A sensor measures physical parameters like temperature, light, pressure, voltage and current. Wireless sensor networks with self-organizing, self-configuring, self-diagnosing and self-healing capabilities have been developed to solve problems or to enable applications that traditional technologies could not address [5]. A major advantage of this kind of networks is low cost, small size and low power consumption. In the field of WSN, the ZigBee technology had met a wide acceptance because of its capability to operate in a large number of applications.

In this paper, the main objective is to study the functionality of a ZigBee based monitoring and supervising system. The investigated PV system is located on the roof of the Technological and Educational Institute of Crete (Chania/Greece). It consists of six (6) polycrystalline-Si technology PV modules and rated power 100 Wpeak each connected in parallel. The remainder of this paper deals with a brief description of the ZigBee standard, a presentation of the developed hardware and the current case study with representative experimental measurements.

\section{Contribution to Sustainability}

Studies on photovoltaic phenomena have drawn the attention to the problem of PV behavior under varying environmental conditions [6]. The major problem is the strong dependence of a PV system response on many extrinsic factors such as temperature, insolation, cloudiness and pollution [7]. Another problem to be solved is to find an efficient and also cost-effective monitoring and supervising method of a PV plant even for small PV systems. Regular performance checks on the functioning of PV systems are necessary for a reliable use and successful integration of a PV. Monitoring for large PV systems is performed by specially designed hardware and software that might be expensive and is mainly operated by specially trained personnel. For small PV systems, up to $10 \mathrm{~kW}$, these checks are not performed due to monitoring system costs [8]. Therefore such small systems are often not checked on a regular basis. This situation can lead to partial energy losses that usual originate from partial system faults or decreasing performance that can be unnoticed for a long time. Thus, in order to achieve the maximum energy out of a PV plant, a low-cost, easy to use monitoring system is needed.

ZigBee technology is a low data rate, low power consumption, low cost, wireless networking protocol targeted towards wireless connections between electronic devices in automation and remote controlling applications [9, 10]. ZigBee can be implemented in mesh networks larger than it is possible with Bluetooth. ZigBee compliant wireless devices are expected to transmit 10-50 meters, depending on the $\mathrm{RF}$ environment and the power output consumption required for a given application [11-14]. 


\section{ZigBee and System Architecture}

The ZigBee standard is built on top of the IEEE 802.15.4 standard. The IEEE 802.15.4 standard defines the physical and MAC (Medium Access Control) layers for low-rate wireless personal area networks $[18,19]$. Other benefits of this standard are energy measurements over the operated channel, Link Quality Indication (LQI), Received Signal Strength Indication (RSSI) and clear channel assessment. Different network topologies are supported like tree, star, peer to peer and mesh. ZigBee is targeted mainly for battery-powered applications where low data rate, low cost, and long battery life are the main requirements. The main field of applications for ZigBee focuses on industrial control, home automation, energy monitoring, wireless logistic systems and many others, as this standard tends to meet wide acceptance from semiconductor companies.

In this study, PV monitoring system architecture consists of two (2) basic blocks; the PV area sector (PVAS) and the central station sector (CSS):

$P V$ area sector: This is actually the PV plant area where basic monitoring parameters are monitored. An alternative title instead of PVAS could be the (ZigBee modules area) and this is because each PV module in the plant is equipped with a ZigBee module and a set of sensors. The PVAS is part of ZigBee end devices (ZED) and a ZigBee router node (ROUT) that serves all ZED's as a sink to the ZigBee coordinator (COO).

Central Station sector: It can be assumed that CSS is the control station of a PV system. In such a station, various components of a PV system like the inverter, the batteries and the battery charger could be found. In the CSS the ZigBee coordinator module, the host PC and the remote measurement board (REMB) are located.

Remote Measurement Board: For safety reasons, all high voltages and currents of the PV plant are monitored in a separate PC board that is connected to the coordinator board.

Furthermore, the monitoring system generally operates as follows:

1. The attached in each PV module ZigBee notes, sends data about their voltage, current and temperature volumes. Data from ZEDs are sent to the router which is also an attached mote to a PV module. The router has the same functionality as with any ZED but is additionally capable of PV array angle reading (when a sun tracking system is used) and has also a dust sensor. All collected data from ZEDs and the router itself are sent to the coordinator board.

2. The coordinator board collects data from ZEDs via the router and supplies the host PC. The host PC runs suitable Graphic User Interface (GUI) software where monitoring and data logging are implemented.

Finally, the coordinator board monitors the total current and voltage originating from the PV plant and inverter as well. Extra functionality such as inverter and battery temperature as well as relative humidity is available. In the following Table 1 a list of all the measured parameters by the developed PV monitoring system are presented. 
Table 1. List of the monitored parameters

\begin{tabular}{llll}
\hline No & Parameter & No & Parameter \\
\hline 1 & Modules output voltage & 8 & Inverter output current \\
2 & Modules output current & 9 & Inverter temperature \\
3 & Modules temperature & 10 & Battery voltage \\
4 & Ambient temperature & 11 & Battery charging current \\
5 & System output voltage & 12 & Battery temperature \\
6 & System output current & 13 & Relative humidity \\
7 & Inverter output voltage & 14 & Dust air concentration
\end{tabular}

\subsection{Coordinator Board}

As described above, the COO board is part of the CSS. The coordinator board is the central component of the monitoring system due to the fact that it is the coordinator of the ZigBee network. The coordinator board that depicted in Fig. 1 hosts the ZigBee module (ETRX2-PA), an Analog to Digital Converter (ADC) (ADC0808), a Universal Serial Bus (USB) to RS-232 interface/converter (CP2102), a battery backed up power supply, a relative humidity sensor (HIH-3041) and an RJ-45 for connection to the REMB.

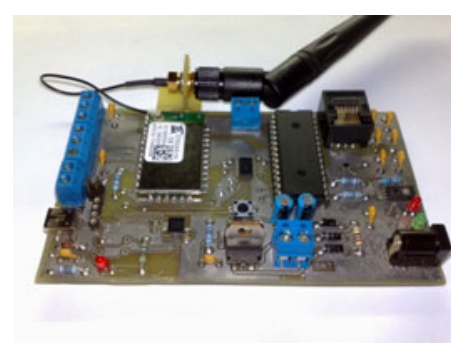

Fig. 1. The coordinator board

A USB port is provided for connection of the COO board to the host PC. Relative humidity levels as well as the control station ambient temperature can be monitored. One extra (LM-35) temp sensor can be connected to the COO board in order to read the inverter temperature and the ambient temperature around the CSS. An external $2.4 \mathrm{GHz}$ antenna ensures enough radio coverage in case where the PVAS is far away from CSS.

\subsection{REMB}

The REMB is the second part of the CSS and it is a board where all sensors are connected as it is shown in Fig. 2. As it is clear from the block diagram, a number of sensors like current and temperature are separated from the REMB. This is mainly because the measurement points (e.g. inverter voltage, current, or temperature) may be located far from REMB. The connection of sensors to the REMB is done through shielded stereo audio cables. 
The reason that the CSS is divided into two parts (COO board and REMB) is mainly because with this implementation, all dangerous high voltages and currents are kept isolated from COO board and REMB where user might have access when the system is on. Other reason is that interferences from high voltages and currents are kept away from the ETRX2-PA module and the PC. Connection between REMB and COO board is done with a CAT5 Ethernet cable via the RJ-45 ports. The power needed for the REMB to operate $(+5 \mathrm{~V})$ is supplied directly from the COO board. In Fig. 3 the developed REMB is depicted.

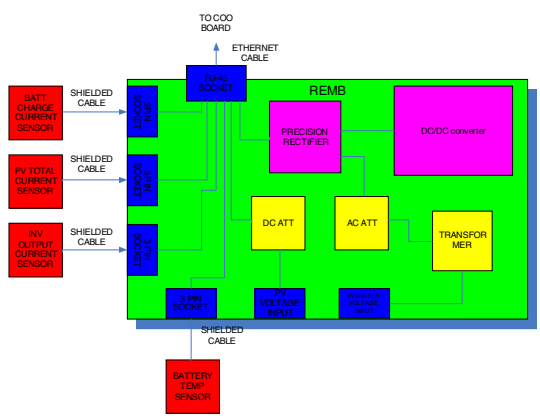

Fig. 2. REMB block diagram

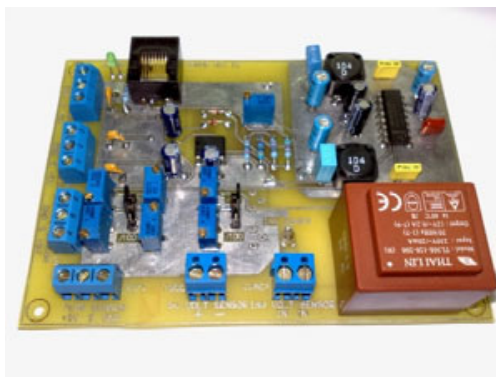

Fig. 3. The developed Remote Measurement Board (REMB)

\subsection{Router}

The router board provides a link between the ZEDs and the COO. The block diagram of the router is shown in Fig. 4. As already described previously, the router serves all end devices as a data sink to the COO board.

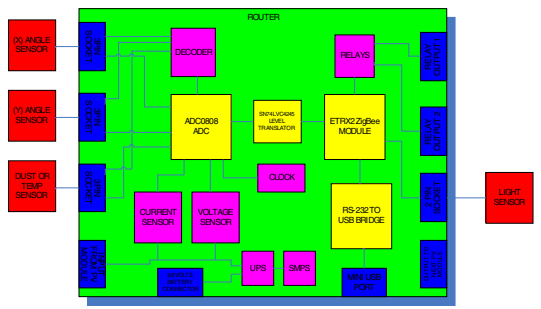

Fig. 4. Router block diagram

The Router has extra functionality compared to end devices. Except current, voltage and temperature, the router can monitor the air dust concentration and also the angle of the PV array in case of a tracking system is used. In addition, the router is equipped with two relay controlled outputs that can be used to control the motors, servos or the actuators of a tracking system and also a set of hall-effect angle sensors that are used to provide feedback to the tracking controller. The router board can also broadcast the sun illumination levels by simply connecting a $\mathrm{Si}$ photodiode or an 
off-the-shelf pyranometer to one of its inputs. To avoid the use of batteries in the router board as well as in ZEDs and in order to operate at low PV panel output voltages (mainly at low insolation levels), a specially designed Switch Mode Power Supply (SMPS) is used. This configuration allows the device to operate normally when the PV module voltage exceeds a three volts $(3 \mathrm{~V})$ threshold. After tests and redesigns, the SMPS/voltage regulator solution became the most efficient and suitable compared to the conventional linear regulated power supplies.

\subsection{ZED}

An end device is the simplest device on this monitoring system. The parameters that are monitored are the PV modules output voltage, current, and temperature. Fig. 5 shows the block diagram of the end device.

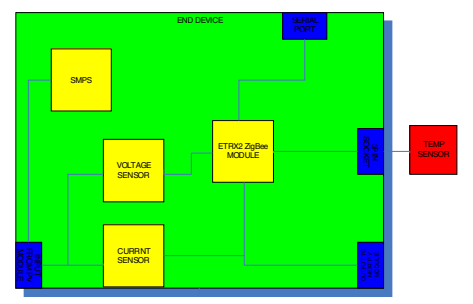

Fig. 5. Block diagram of a ZED

\section{Measurements and Experiments}

Two different experiments were conducted in order to verify the performance and reliability of the ZigBee PV monitoring system.

\section{i. Bad connection experiment}

In the first experiment, the case of a bad or corrupted connection has been simulated. In case $\mathrm{N}_{\mathrm{o}} 1$, we disconnect the positive terminal of a PV module. This disconnection led to a zero current reading of the respective PV module (PV module $\mathrm{n}_{\mathrm{o}} 3$ ) in the GUI application. The absence of current measurement but the presence of a $17 \mathrm{~V}$ voltage reading leads to the conclusion that the PV module is disconnected from the DC bus. Such a state can be easily managed by a software application in order to enable an alarm. In case $\mathrm{N}_{\mathrm{o}} 2$, we connected a PV module (PV module $\mathrm{n}_{0} 4$ ) in the DC bus but in that case corroded connectors used. This situation led to fluctuations of current and voltage readings in the GUI for the specific PV module confirming the bad connection.

\section{ii. Low performance of a PV module}

In this experiment, the case of a low performance caused by dirt or dust in the transparent surface of the module had been investigated. A mixture of fine sand and water had been sprayed to the glass surface of $\mathrm{PV} \mathrm{N}_{0} 4$ simulating the red rain effect caused from the African dust, that is a very common phenomenon in the area of Crete. The PV monitor displayed a slight lower current reading in comparison to the mean value of the rest PV modules. The dust sensor discussed previously can alert for high 
air dust concentration. Depending on the software extra reading can be extracted like power (W) and energy (Joules).

In Fig. 6 a number of graphs are shown representing the measurements corresponding to a specific day for each PV panel separately. The small differences between the graphs are mostly sensor errors. Another reason of these variations can be the fact that none of the PV module outputs the same amount of energy for a specified sun illumination.

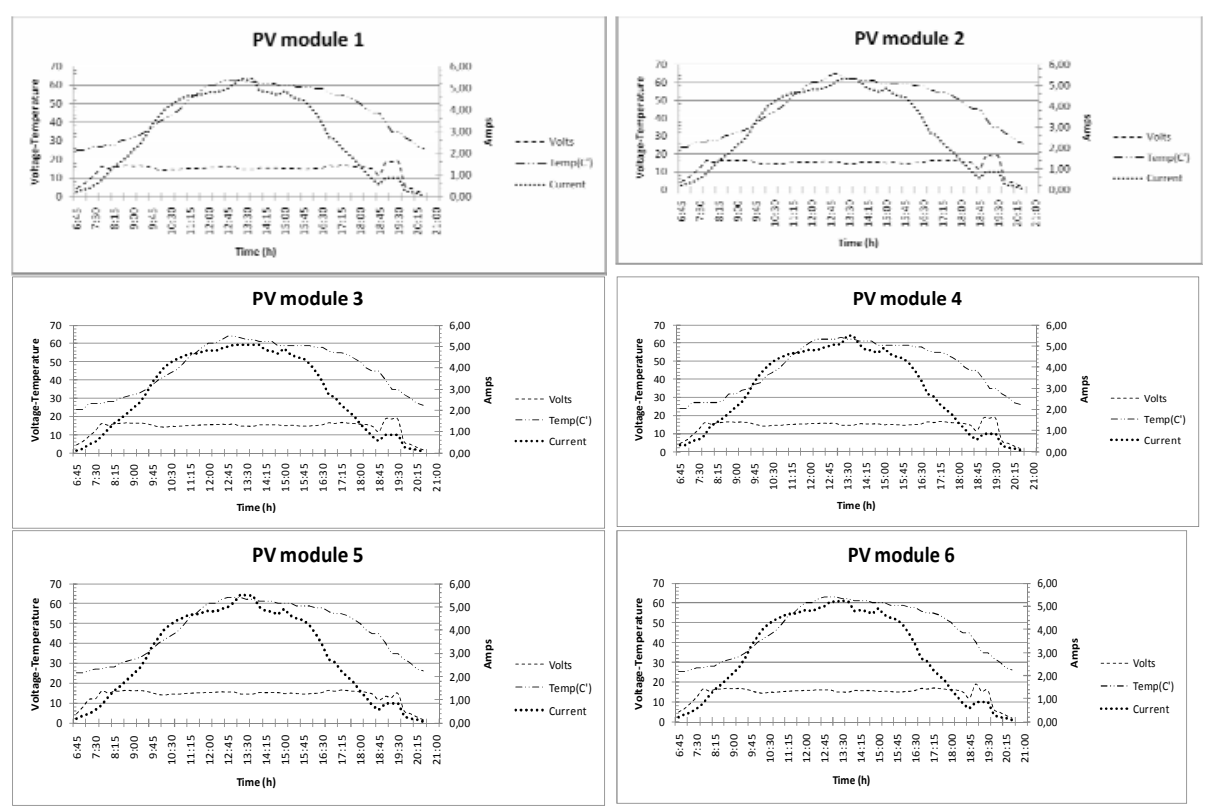

Fig. 6. PV current, voltage and temperature measurements of a specific day

\section{Conclusions}

In this paper, the prospect of using the ZigBee wireless technology in the field of PV supervising and monitoring was experimentally assessed. The system has been successfully tested on a low power PV system consisting of six (6) Photovoltaics modules (100Wp each) located at the main TEIC building (Chania/Greece). This kind of system can be installed in any kind of PV generation system regardless of size, as the ZigBee standard allows a very large volume of nodes (up to approximately $65,000)$ to be connected. The system presented here, provides accurate and also real time information about not only the overall PV plant behavior but also for any PV module alone.

Failures as well as disoperation of any component that consist the PV system can be identified immediately. As it is previously described, the system estimates and monitors the state of the PV plant through a strain forward process contrary to other systems that base their operation on indirectly methods such as complicated statistical 
algorithms and comparisons of current performances with previous. In future works, connection of the system to the internet can be implemented in order for the user to observe the PV plant status from his personal computer. In case of no internet line availability, a GSM modem or other means of broadcast can be easily used.

Another important factor that can be characterized is the fact that here is no need of system inspection in the case of malfunctioning. Thus, specially trained personnel (maintenance operator) costs and also energy could be saved by the fact that the malfunctioning element is located very fast. Finally, the issue of integrating a ZigBee monitoring mote in each PV module in order to produce ready of-the-shelf ZigBee build in PV modules must be seriously investigated by the PV module manufacturers.

\section{References}

1. Gagliarducci, M., Lampasi, D.A., Podesta, L.: GSM-based monitoring and control of photovoltaic power generation. Journal of Measurement 40, 314-321 (2007)

2. Kalaitzakis, K., Koutroulis, E., Vlachos, V.: Development of a data acquisition system for remote monitoring of renewable energy systems. Journal of Measurement 34, 75-83 (2003)

3. Koutroulis, E., Kalaitzakis, K.: Development of an integrated data-acquisition system enewable energy sources systems monitoring. Journal of Renewable Energy 28, 139-152 (2003)

4. Koizumi, H., Mizuno, T.: A novel micro controller for grid-connected photovoltaic systems. IEEE Trans. Ind. Electron 53(6), 1889-1897 (2006)

5. Gungor, V.C., Hancke, G.P.: Industrial Wireless Sensor Networks: Challenges, Design Principles, and Technical Approaches. IEEE Transaction on Industrial Electronics 56(10), 4258-4265 (2009)

6. Andrei, H., Dogaru-Ulieru, V., Chicco, G., Cepisca, C., Spertino, F.: Photovoltaic applications. Journal of Materials Processing Technology 181, 267-273 (2007)

7. Vergura, S., Acciani, G., Amoruso, V., Patrono, G.E., Vacca, F.: Descriptive and Inferential Statistics for Supervising and Monitoring the Operation of PV Plants. IEEE Transaction on Industrial Electronics 56(11), 4456-4464 (2009)

8. Drews, A., de Keizer, A.C., Beyer, H.G., Lorenz, E., Betcke, J., van Sark, W.G.J.H.M., et al.: Monitoring and remote failure detection of grid connected PV systems based on satellite observations. Journal of solar energy 81, 548-564 (2007)

9. Kay, R., Mattern, F.: The Design Space of Wireless Sensor Networks. IEEE Wireless Communications 11(6), 54-61 (2004)

10. Haenselmann, T.: Sensornetworks. GFDL Wireless Sensor Network Textbook (2006)

11. Chong, C., Kumar, S.P.: Sensor Networks: Evolution, Opportunities, and Challenges. Proc. IEEE 91(8), 1247-1256 (2003)

12. Culler, D., Estrin, D., Srivastava, M.: Overview of Sensor Networks. Computer 37(8), 41-49 (2004)

13. Jiang, Q., Manivannan, D.: Routing Protocols for Sensor Networks. In: Proc. 1st IEEE Consumer Comm. and Networking Conf., pp. 93-98. IEEE Press, Los Alamitos (2004)

14. Labiod, H., Afifi, H., Santis, C.: WI-FI, Bluetooth, ZigBee and Wimax. Springer, Netherland (2007) 